\title{
Improved prediction of incident vertebral fractures using opportunistic QCT compared to DXA
}

\author{
Maximilian T. Löffler ${ }^{1}$ (D) Alina Jacob ${ }^{1}$ - Alexander Valentinitsch ${ }^{1} \cdot$ Anna Rienmüller $^{2,3}$ • Claus Zimmer ${ }^{1}$. \\ Yu-Mi Ryang ${ }^{2} \cdot$ Thomas Baum $^{1} \cdot$ Jan S. Kirschke ${ }^{1}$
}

Received: 22 August 2018 / Revised: 18 December 2018 / Accepted: 17 January 2019 / Published online: 21 February 2019

(C) The Author(s) 2019

\begin{abstract}
Objectives To compare opportunistic quantitative CT (QCT) with dual energy X-ray absorptiometry (DXA) in their ability to predict incident vertebral fractures.

Methods We included 84 patients aged 50 years and older, who had routine CT including the lumbar spine and DXA within a 12-month period (baseline) as well as follow-up imaging after at least 12 months or who sustained an incident vertebral fracture documented earlier. Patients with bone disorders aside from osteoporosis were excluded. Fracture status and trabecular bone mineral density (BMD) were retrospectively evaluated in baseline $\mathrm{CT}$ and fracture status was reassessed at follow-up. $\mathrm{BMD}_{\mathrm{QCT}}$ was assessed by opportunistic QCT with asynchronous calibration of multiple MDCT scanners.

Results Sixteen patients had incident vertebral fractures showing lower mean $\mathrm{BMD}_{\mathrm{QCT}}$ than patients without fracture $(p=0.001)$. For the risk of incident vertebral fractures, the hazard ratio increased per $\mathrm{SD}$ in $\mathrm{BMD}_{\mathrm{QCT}}(4.07 ; 95 \% \mathrm{CI}, 1.98-8.38)$, as well as after adjusting for age, sex, and prevalent fractures $(2.54 ; 95 \%$ CI, 1.09-5.90). For DXA, a statistically significant increase in relative hazard per SD decrease in $T$-score was only observed after age and sex adjustment (1.57; 95\% CI, 1.04-2.38). The predictability of incident vertebral fractures was good by $\mathrm{BMD}_{\mathrm{QCT}}(\mathrm{AUC}=0.76 ; 95 \% \mathrm{CI}, 0.64-0.89)$ and non-significant by $T$-scores. Asynchronously calibrated CT scanners showed good long-term stability (linear drift ranging from -0.55 to $-2.29 \mathrm{HU}$ per year). Conclusions Opportunistic screening of mainly neurosurgical and oncologic patients in CT performed for indications other than densitometry allows for better risk assessment of imminent vertebral fractures than dedicated DXA.

Key Points

- Opportunistic QCT predicts osteoporotic vertebral fractures better than DXA reference standard in mainly neurosurgical and oncologic patients.

- More than every second patient (56\%) with an incident vertebral fracture was misdiagnosed not having osteoporosis according to DXA.

- Standard ACR QCT-cutoff values for osteoporosis $\left(<80 \mathrm{mg} / \mathrm{cm}^{3}\right)$ and osteopenia $\left(\leq 120 \mathrm{mg} / \mathrm{cm}^{3}\right)$ can also be applied scanner independently in calibrated opportunistic QCT.
\end{abstract}

Keywords Bone density $\cdot$ Osteoporosis $\cdot$ Spinal fractures $\cdot$ Photon absorptiometry $\cdot$ Multidetector computed tomography

Maximilian T. Löffler

m_loeffler@web.de

1 Department of Neuroradiology, Klinikum rechts der Isar, Technische Universität München, Ismaninger Str. 22, 81675 Munich, Germany

2 Department of Neurosurgery, Klinikum rechts der Isar, Technische Universität München, Munich, Germany

3 Department of Orthopedic and Trauma Surgery, Medical University Vienna, Vienna, Austria

\author{
Abbreviations \\ ACR American College of Radiology \\ BMD Bone mineral density \\ CI $\quad 95 \%$ confidence interval \\ $\mathrm{CV}$ Coefficient of variation of the standard error of the \\ estimate \\ DXA Dual energy X-ray absorptiometry \\ HA Hydroxyapatite \\ HR Hazard ratio \\ QCT Quantitative CT \\ SD Standard deviation \\ SL Slope of linear regression
}




\section{Introduction}

Osteoporosis is a metabolic bone disease leading to reduced bone strength and manifesting in low-energy fractures [1]. Resulting pain and disability pose a huge burden on patients and society [2, 3]. Effective prevention and medical treatment for osteoporosis exist [4], but are not initiated in many patients $[3,5]$, partly because bone densitometry is under-utilized [6, 7]. Bone mineral density (BMD) as the single most important parameter accounts for approximately $70 \%$ of bone strength [8].

For the diagnosis of osteoporosis, the up-to-date reference standard in clinical bone densitometry is dual energy X-ray absorptiometry (DXA) [9]. This projectional technique is performed at the spine and hip in order to formulate a diagnosis based on a WHO normative population [10]. However, the role of DXA in the diagnosis of osteoporosis can be put into question, given that in a large population-based study, less than half of women (44\%) and even fewer men (21\%) of all individuals with prevalent osteoporotic fractures were correctly diagnosed with osteoporosis by DXA [11].

Quantitative CT (QCT) is a notable alternative to DXA with at least the same ability to predict vertebral fractures in women, although it is not officially approved to diagnose osteoporosis, since the WHO classification relies on $T$ scores derived by DXA [12]. QCT is a non-projectional technique performed on clinical CT scanners to measure volumetric BMD. Due to its three-dimensional characteristic, QCT is largely independent of degenerative changes in the spine and can differentiate between cortical and trabecular bone. Trabecular bone is about eight times more metabolically active than cortical bone and therefore prone to changes in osteoporosis [12]. Osteodensitometry in routine CT scans, which have been acquired for other purposes, can distinguish osteoporotic from healthy individuals [13] and bears a huge potential of opportunistic screening [14]. Accordingly, densitometry based on non-dedicated CT scans is named opportunistic QCT. In the following, "BMD" will refer to volumetric BMD as assessed by opportunistic QCT - not DXA measured areal BMD - as previously encouraged [12]. Wherever helpful for the reader to avoid confusion, we explicitly identify $\mathrm{BMD}_{\mathrm{QCT}}$ as being derived from $\mathrm{CT}$ measurements.

The comparative potential of QCT and DXA to discriminate between patients with and without prevalent vertebral fractures has been investigated in many cross-sectional studies [15-18]. Recently, the risk of future vertebral fractures has been investigated in opportunistic CT data [19]; however, DXA data was not included. In the present study, we investigate the association between the risk of future osteoporotic vertebral fractures and opportunistic BMD measurements in routine $\mathrm{CT}$ scans acquired for other purposes compared to measurements of the reference standard DXA.

\section{Methods}

\section{Study population}

The local institutional review board approved this retrospective study and waived written informed consent. In a formal query on the institutional database including all patients registered until May 2017, we identified 325 patients aged 50 years and older at DXA, who had DXA and baseline CT including the lumbar spine within 12 months. After excluding patients for several reasons (history of vertebral metastasis or hematologic disorder [16], CT on a scanner without calibration or with different tube voltage setting [10], and CT without at least one lumbar vertebra assessable for densitometry [6]), 84 patients with follow-up imaging after at least 12 months or who sustained an incident vertebral fracture documented in an earlier imaging study were included. These patients had routine $\mathrm{CT}$ for a variety of indication other than densitometry (36\% acute back pain or suspected spinal fracture; $30 \%$ staging, restaging, or malignoma follow-up; $15 \%$ exclusion of acute abdominal pathology; $12 \%$ chronic back pain; and 7\% postoperative $\mathrm{CT}$ after neurosurgery). An incident vertebral fracture was defined as a fracture that occurred either in a previously non-fractured vertebra (Fig. 1) or in an already fractured, consolidated vertebra with increase in at least one grade of the semiquantitative scale by Genant [20]. Consolidation was ensured by the absence of bone marrow edema in recent MR imaging. Active, progressive vertebral fractures (with bone marrow edema in MRI or signs of callus formation in CT [21]) were not considered as incidental fractures as they are usually associated with continuous clinical symptoms [22].

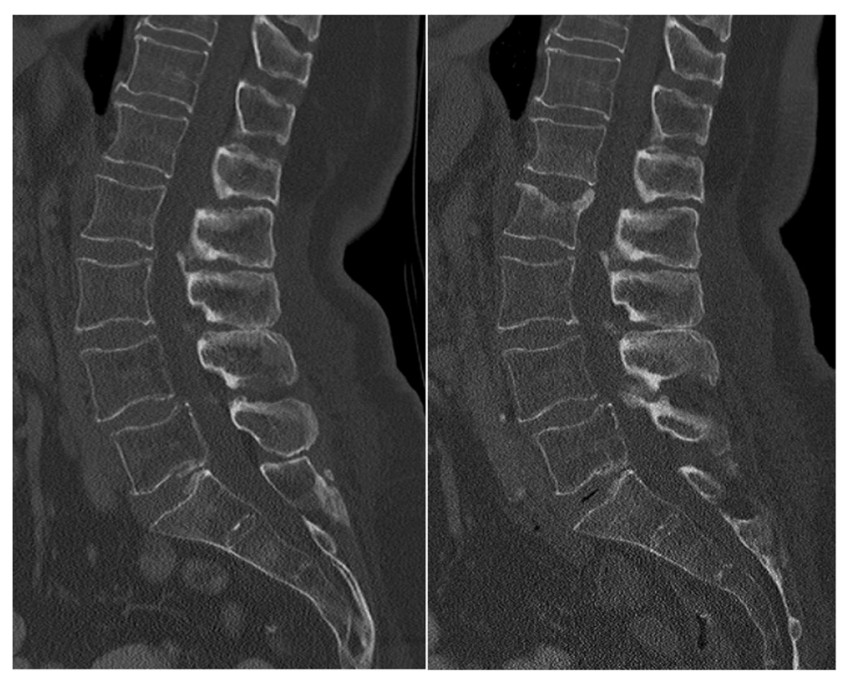

Fig. 1 Left: baseline CT of a 72-year-old female patient with osteopenia according to DXA $(T=-1.7)$ and osteoporosis according to opportunistic QCT $\left(\mathrm{BMD}=70.5 \mathrm{mg} / \mathrm{cm}^{3}\right)$. Right: in follow-up after 5.2 years, the patient had sustained an incident vertebral compression fracture of $\mathrm{L} 2$ 


\section{Dual energy X-ray absorptiometry}

DXA measurements were performed on a single densitometer (GE Lunar Prodigy, GE Healthcare) by trained technologists and quality was assured through evaluation by experienced physicians supervised by a certified densitometrist. Total proximal femur of both hips and lumbar vertebrae L1 to L4 was assessed in anterior-posterior projection [23]. Those skeletal sites affected by severe local structural change or artifact were excluded. If only one vertebra remained after exclusion of other vertebrae, the measurement was solely based on the hip. The overall lowest $T$-score at the lumbar spine or total proximal femur was reported and accounted for a single diagnosis of osteoporosis [24]. Osteoporosis was defined as $T \leq-2.5$ standard deviations (SD), osteopenia as $-2.5<\mathrm{T} \leq-1 \mathrm{SD}$ [9].

\section{Computed tomography}

Baseline CT was performed on five multidetector computed tomography (MDCT) scanners in the same hospital (Philips Brilliance 64 and iCT 256, Philips Medical Care; Siemens Somatom Definition AS+, Definition AS, and Sensation Cardiac 64, Siemens Healthineers), partly with administration of oral (Barilux Scan, Sanochemia Diagnostics) and intravenous contrast medium (Imeron 400, Bracco). Image data was acquired in helical mode with a peak tube voltage of $120 \mathrm{kVp}$, a slice thickness of 0.9 to $1 \mathrm{~mm}$ and adaptive tube load. Sagittal reformations with a slice thickness of $2 \mathrm{~mm}$ and standard bone kernel were reconstructed, as proposed for better fracture detection [25].

\section{Opportunistic QCT}

Asynchronous QCT was performed in baseline CT, a technique that provides results comparable to conventional QCT [26]. Attenuation values in HU were manually sampled with tools of the institutional picture archiving and communication system software (Sectra IDS7, Sectra AB) and transformed into volumetric BMD with conversion equations calculated by asynchronous calibration. An experienced radiologist placed a circular region of interest in trabecular bone of lumbar vertebrae L1 to L4, as previously described [27], using on-the-fly calculated midsagittal stacks of $15-\mathrm{mm}$ thickness. Sampled HU was averaged over assessed vertebrae, omitting fractured vertebra or those with apparent alterations of the trabecular bone due to degeneration or hemangioma.

HU-to-BMD conversion equations were calculated by linear regression, in three scanners (Philips Brilliance 64, iCT 256, and Siemens Somatom Definition AS+) based on measurements of density-reference phantoms (QRM) in dedicated scans with the same tube voltage and scanner settings as in clinical routine acquisitions, and in two already decommissioned scanners (Siemens Somatom Definition AS and Sensation Cardiac 64) based on retrospective measurements of a density-reference phantom (Osteo Phantom, Siemens Healthineers), which had been included in the scanner couch during clinical CT scans for a certain period of time in the past (Fig. 2). Retrospective measurements of the Siemens Osteo phantom and a second calibration phantom (Mindways Software) were performed in CT exams, which were randomly selected from the institutional database in 2-month intervals over the entire time period when phantoms were present. Thereby, long-term scanner stability was evaluated in three scanners (Philips iCT 256, Siemens Somaton Definition AS, and Sensation Cardiac 64). Conversion equations and long-term stability measures are shown in Table 4. A BMD correction offset for contrast-enhanced CT scans with arterial $\left(-8.6 \mathrm{mg} / \mathrm{cm}^{3}\right)$ and portal venous contrast phase $\left(-15.8 \mathrm{mg} / \mathrm{cm}^{3}\right)$ was added based on previous investigations [28]. Osteoporosis was defined as BMD $<80 \mathrm{mg} /$ $\mathrm{cm}^{3}$ and osteopenia as $80 \leq \mathrm{BMD} \leq 120 \mathrm{mg} / \mathrm{cm}^{3}$ [29].

\section{Statistical analysis}

Baseline characteristics were compared using $t$ test for continuous variables and chi-square test for categorical variables. In Cox proportional hazard models, hazard ratio (HR) and $95 \%$ confidence interval (CI) for the risk of incident vertebral fractures were calculated, firstly with unadjusted $\mathrm{BMD}_{\mathrm{QCT}}$ and

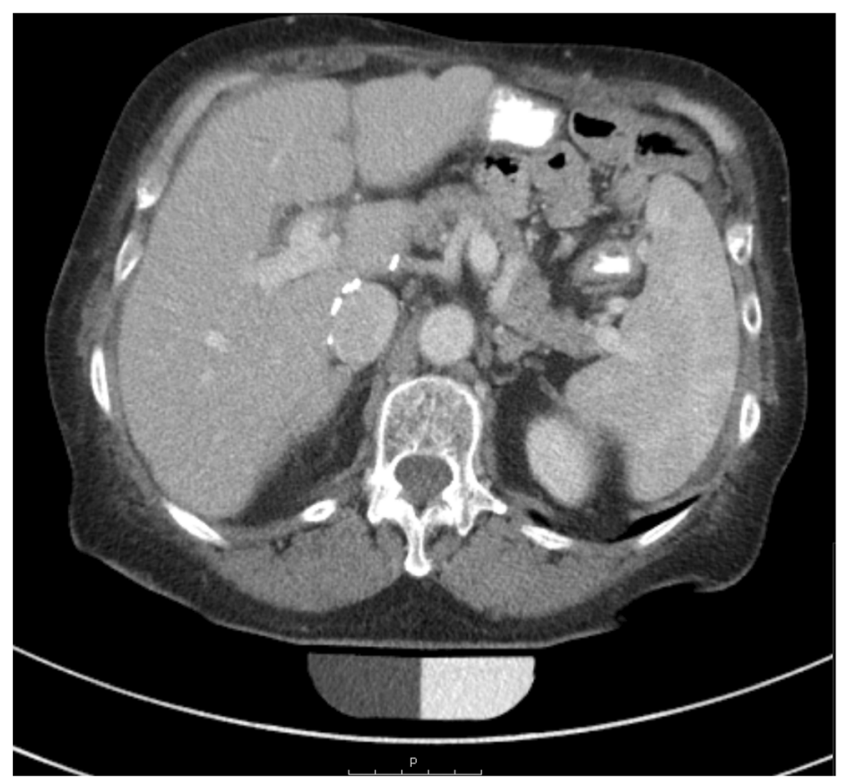

Fig. 2 Routine CT scan of a 63-year-old female patient for follow-up purpose after metastatic gastric cancer and liver transplant with administration of oral and intravenous contrast medium in portal venous phase. For two MDCT scanners (Siemens Somatom Definition AS [in this example] and Sensation Cardiac 64), retrospective measurements of an in-plane calibration phantom present underneath patients during routine scans were used for asynchronous calibration and evaluation of long-term scanner stability 
DXA $T$-score, and secondly with age at DXA, sex, and prevalent fractures as covariates. For better comparability, HR is expressed per SD decrease in $\mathrm{BMD}_{\mathrm{QCT}}$ or DXA T-score. In Kaplan-Meier curves, fracture-free time periods were visualized for patients with osteoporosis, osteopenia, or normal bone density defined either by $\mathrm{BMD}_{\mathrm{QCT}}$ or DXA $T$-score. In ROC analysis, AUC was calculated to predict incident vertebral fractures by $\mathrm{BMD}_{\mathrm{QCT}}$ and DXA T-scores. In order to assess long-term scanner stability, slope of linear regression (SL) and coefficient of variation of the standard error of the estimate (CV) were calculated for measurements of two phases of the calibration phantoms. For each scanner, CV was averaged by the root-mean-square [30]. All statistical analyses were conducted with IBM SPSS Statistics 25 (IBM), with an $\alpha$-level of significance $p<0.05$.

\section{Results}

Over a median follow-up of 2.6 years (interquartile range 1.73.6 years), 16 of 84 patients (19\%) sustained an incident vertebral fracture (Table 1). Patients with incident vertebral fracture were significantly older with a mean age of $73.9 \pm 7.4$ years and had a lower mean $\mathrm{BMD}_{\mathrm{QCT}}$ of $56.7 \pm 31.6 \mathrm{mg} / \mathrm{cm}^{3}$ than patients without fracture with a mean age of $67.7 \pm 8.6$ years $(p=0.01)$ and a mean BMD $_{\mathrm{QCT}}$ of $93.3 \pm 41.7 \mathrm{mg} / \mathrm{cm}^{3}(p=$ 0.001). The number of patients defined as osteoporotic by $\mathrm{BMD}_{\mathrm{QCT}}$ differed significantly between patients with and without incident vertebral fractures $(p=0.004)$. However, there was no significant difference in DXA $T$-score between patients with and without incident vertebral fractures $(p=0.179)$. Seven of 16 patients $(44 \%)$ with incident vertebral fractures were correctly diagnosed with osteoporosis according to DXA, whereas 13 of $16(81 \%)$ were categorized having osteoporotic $\mathrm{BMD}_{\mathrm{QCT}}$.

There was a statistically significant association between the risk of incident vertebral fractures and unadjusted trabecular BMD with a HR of 4.07 per SD decrease in $\mathrm{BMD}_{\mathrm{QCT}}(\mathrm{CI}$, 1.98-8.38); there was no significant association with unadjusted DXA T-score (Table 2, Figs. 3 and 4). The HR for incident vertebral fractures per SD decrease in $\mathrm{BMD}_{\mathrm{QCT}}$ varied, but remained statistically significant, after adjusting for age (3.60; CI, 1.70-7.64), for age and sex (4.02; CI, 1.838.82 ), and for age, sex, and prevalent fractures (2.54; CI, 1.09 5.90). Only after adjusting for age and sex, a statistically significant increase in HR per SD decrease in DXA $T$-score was observed (1.57; CI, 1.04-2.38). $\mathrm{BMD}_{\mathrm{QCT}}$ was a significant classifier to predict incident vertebral fractures $(\mathrm{AUC}=0.76$; CI, 0.64-0.89), DXA T-score was not (Table 3 and Fig. 5). $\mathrm{BMD}_{\mathrm{QCT}}$ values greater than or equal to $79.6 \mathrm{mg} / \mathrm{cm}^{3}$ could predict incident vertebral fracture with a specificity of $81 \%$ and a sensitivity of $59 \%$.

Long-term scanner stability was good for all three investigated MDCT scanners. Linear drift was $S L_{i C T}=-0.55 \mathrm{HU}$ per year over an observation period of 5.33 years with a $C V_{i C T}=1.1 \%$ for Philips iCT 256, $S L_{A S}=-2.29 \mathrm{HU}$ per year over an observation period of 4 years with a $C V_{A S}=1 \%$ for

Table 1 Baseline characteristics of patients with and without incident vertebral fractures

\begin{tabular}{|c|c|c|c|c|c|}
\hline & & $\begin{array}{l}\text { No incident vertebral } \\
\text { fracture }(n=68)\end{array}$ & $\begin{array}{l}\text { Incident vertebral } \\
\text { fracture }(n=16)\end{array}$ & $\begin{array}{l}\text { No vs. incident } \\
\text { vertebral fracture }\end{array}$ & All $(n=84)$ \\
\hline \multicolumn{2}{|l|}{ Women, $n(\%)$} & $54(79 \%)$ & $13(81 \%)$ & n.s. & $67(80 \%)$ \\
\hline \multicolumn{2}{|l|}{ Age at DXA, mean (SD) } & $67.7(8.6)$ & $73.9(7.4)$ & $p=0.01$ & $68.9(8.7)$ \\
\hline \multicolumn{2}{|c|}{ Days between DXA and CT, median (range) } & $70(0-362)$ & $34(0-350)$ & n.s. & $62(0-362)$ \\
\hline \multicolumn{2}{|c|}{ Days to follow-up imaging, median (range) } & $1018(373-2425)$ & $768(19-1891)$ & $p=0.049$ & $935(19-2425)$ \\
\hline \multicolumn{2}{|l|}{ Non-enhanced CT scans, $n(\%)$} & $33(49 \%)$ & $7(44 \%)$ & n.s. & $40(48 \%)$ \\
\hline \multicolumn{2}{|l|}{ Diagnosis by lumbar DXA, $n(\%)$} & $39(57 \%)$ & $10(63 \%)$ & n.s. & $49(58 \%)$ \\
\hline \multicolumn{2}{|l|}{ BMD by QCT, mean (SD) } & $93.3(41.7)$ & $56.7(31.6)$ & $p=0.001$ & $86.3(42.4)$ \\
\hline \multicolumn{2}{|l|}{ DXA $T$-score, mean (SD) } & $-1.6(1.7)$ & $-2.2(1.8)$ & n.s. & $-1.7(1.7)$ \\
\hline \multirow{4}{*}{$\begin{array}{l}\text { Maximum Genant grade of prevalent } \\
\quad \text { fractures, } n(\%)\end{array}$} & No fracture & $35(51 \%)$ & $4(25 \%)$ & n.s. & $39(46 \%)$ \\
\hline & Grade 1 & $10(15 \%)$ & $1(6 \%)$ & n.s. & $11(13 \%)$ \\
\hline & Grade 2 & $12(18 \%)$ & $4(25 \%)$ & n.s. & $16(19 \%)$ \\
\hline & Grade 3 & $11(16 \%)$ & $7(44 \%)$ & $p=0.016$ & $18(22 \%)$ \\
\hline \multirow[t]{3}{*}{ Bone density by QCT, $n(\%)$} & Normal & $15(22 \%)$ & $1(6 \%)$ & n.s. & $16(19 \%)$ \\
\hline & Osteopenia & $25(37 \%)$ & $2(13 \%)$ & n.s. & $27(32 \%)$ \\
\hline & Osteoporosis & $28(41 \%)$ & $13(81 \%)$ & $p=0.004$ & $41(49 \%)$ \\
\hline \multirow[t]{3}{*}{ Bone density by DXA, $n(\%)$} & Normal & $22(32 \%)$ & $2(12 \%)$ & n.s. & $24(29 \%)$ \\
\hline & Osteopenia & $24(35 \%)$ & $7(44 \%)$ & n.s. & $31(37 \%)$ \\
\hline & Osteoporosis & $22(33 \%)$ & $7(44 \%)$ & n.s. & $29(34 \%)$ \\
\hline
\end{tabular}

$S D$, standard deviation; n.s., non-significant at the $\alpha$-level $p<0.05$ 
Table 2 Uni- and multivariate (adjusted for age at DXA, sex, and prevalent vertebral fractures) hazard ratios for the risk of incident vertebral fractures

\begin{tabular}{lllll}
\hline Main variable & \multicolumn{4}{l}{ Hazard ratio per SD decrease in $T$-score/BMD $(\mathrm{CI})$} \\
\cline { 2 - 5 } & Unadjusted & Adjusted for & & \\
\cline { 2 - 5 } & & Age & Age and sex & Age, sex and prevFX \\
\hline T-score by DXA & $1.36(0.93-1.99)$ & $1.43(0.98-2.09)$ & $1.57(1.04-2.38)$ & $1.55(0.97-2.48)$ \\
BMD by QCT & $4.07(1.98-8.38)$ & $3.60(1.70-7.64)$ & $4.02(1.83-8.82)$ & $2.54(1.09-5.90)$ \\
\hline
\end{tabular}

Statistically significant hazard ratios are in italics. $C I, 95 \%$ confidence interval; $S D$, standard deviation; prev $F X$, maximum grade of prevalent vertebral fractures according to semiquantitative score by Genant
Siemens Somatom Definition AS, and $S L_{C 64}=-0.81 \mathrm{HU}$ per year over an observation period of 4.09 years with a $C V_{C 64}=$ $0.7 \%$ for Siemens Somatom Sensation Cardiac 64 (Table 4).

\section{Discussion}

In this retrospective study, trabecular BMD assessed by opportunistic QCT showed a high association with the risk of incident vertebral fractures in a mixed population of mainly neurosurgical and oncologic patients. In contrast, the association of $T$-scores measures by DXA was non-significant. Only after adjusting for age and sex, $T$-scores were associated with the risk of incident vertebral fracture. Furthermore, more than every second patient (56\%) who developed a new osteoporotic vertebral fracture was not diagnosed with osteoporosis according to DXA, whereas the rate of false-negative diagnosis by opportunistic QCT was much lower (19\%).

Many cross-sectional studies compared the capability of DXA and conventional QCT to discriminate between patients with and without prevalent spinal fractures [15-18, 31, 32]. A better ability of opportunistic QCT than DXA to classify these patients was suggested as a secondary result in a study, in which 22 out of 37 patients with a prevalent vertebral fracture (59\%) had non-osteoporotic DXA T-scores [33]. There are further reports when DXA struggled to correctly diagnose approximately every second patient with manifest osteoporosis $[34,35]$. The influence of degenerative changes of the spine on the results of DXA is a long known issue [36-38], that can be mostly overcome by QCT $[34,39,40]$. It seems plausible that in our study population the diagnosis of osteoporosis by DXA was less accurate than by QCT, because there was a majority of elderly neurosurgical patients presenting themselves with back pain and most likely showing a degree of spinal degeneration above average.

Longitudinal studies reporting future vertebral fractures are rare, mostly using dedicated quantitative or biomechanical CT in prospective cohorts [41-43], and/or lacking reference DXA scans of the spine [19, 43]. To the best of our knowledge, no study has been conducted comparing non-dedicated (=opportunistic) QCT with DXA regarding the association with the risk of future vertebral fractures. Our results are in accordance
Fig. 3 Kaplan-Meier curves of time periods without an incident vertebral fracture stratified by opportunistic QCT into patients with normal $\left(>120 \mathrm{mg} / \mathrm{cm}^{3}\right)$, osteopenic $\left(80 \leq \mathrm{BMD} \leq 120 \mathrm{mg} / \mathrm{cm}^{3}\right)$, or osteoporotic BMD $\left(<80 \mathrm{mg} / \mathrm{cm}^{3}\right)$

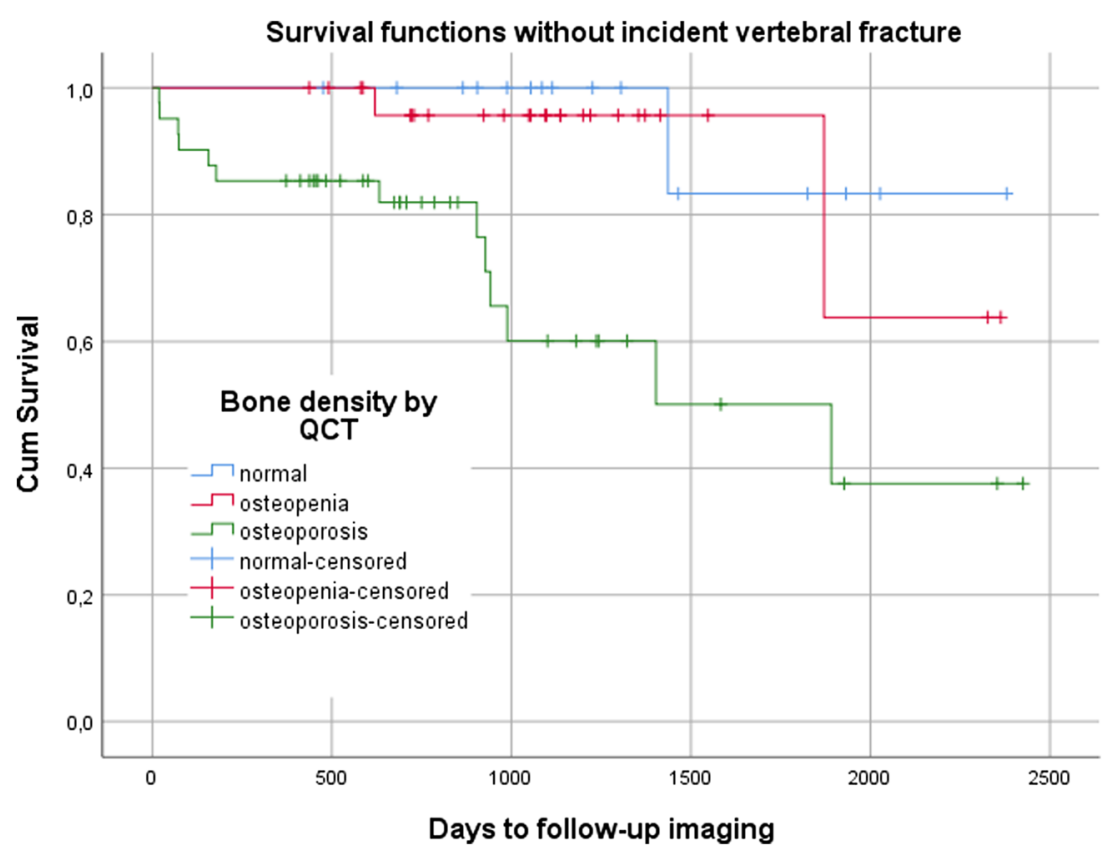


Fig. 4 Kaplan-Meier curves of time periods without an incident vertebral fracture stratified by DXA into patients with normal bone mass $(\mathrm{T}>-1)$, osteopenia $(-2.5<\mathrm{T} \leq-1)$, or osteoporosis $(\mathrm{T} \leq-2.5)$

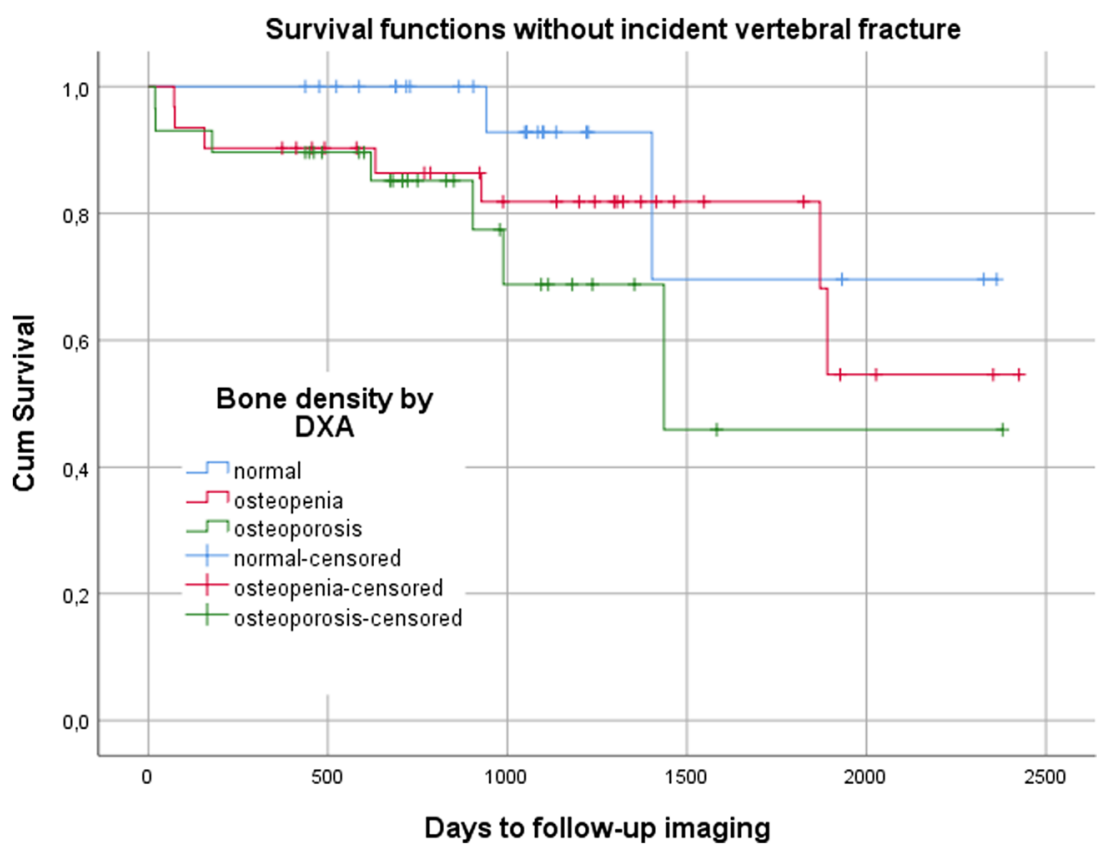

with previous findings of similar longitudinal studies. Analyzing the prospective database of men aged 65 years and older (MrOS), a higher association with the risk of new clinical vertebral fractures was found for integral BMD measured by QCT than for areal BMD measured by DXA [41]. The age-adjusted relative hazard for new clinical vertebral fractures increased by 5.7 per SD decrease in integral BMD at the spine, and by 3.2 and 1.8 per SD decrease in areal BMD at the lumbar spine and femoral neck, respectively. Of note, integral volumetric BMD summarizes trabecular and cortical bone in a similar way to areal BMD, but stays independent of bone size and degenerative alterations. A study on the same prospective cohort (MrOS) found an age- and race-adjusted HR of 3.69 for the prediction of clinical fractures of the spine by trabecular BMD by QCT [42]. Areal BMD by DXA at the lumbar spine had also a strong association with the risk of these fractures $(\mathrm{HR}=3.57)$, but DXA at the femoral neck performed similar to our results $(\mathrm{HR}=1.95)$. In $\mathrm{ROC}$ analysis, trabecular BMD performed also better $(\mathrm{AUC}=0.79)$ than areal BMD at the spine (AUC $=0.72$ ). In our study, more diagnoses $(58 \%)$ were based on DXA measurements at the lumbar spine. This could explain why the predictive performance of
DXA was substantially worse in both aforementioned statistical measures (age- and sex-adjusted $\mathrm{HR}=1.57, \mathrm{AUC}=0.63$ ). As discussed above, this might be due to a selection bias towards neurosurgical patients with above-average spinal degeneration limiting the capabilities of DXA in our population. Recently, the first longitudinal study using opportunistic CT data of multiple scanners without calibration found that L1 vertebral trabecular attenuation blow $90 \mathrm{HU}$ was a significant indicator of decreased fracture-free survival [19].

A $\mathrm{BMD}_{\mathrm{QCT}}$ cutoff predictive for incident vertebral fractures with $81 \%$ specificity (59\% sensitivity), found in our data, closely matched the threshold suggested to be equivalent to the WHO diagnostic category for osteoporosis $\left(<80 \mathrm{mg} / \mathrm{cm}^{3}\right)$ [44, 29]. Predefined thresholds can only be used if CT attenuation values are calibrated to a density-reference phantom usually with known hydroxyapatite (HA) concentrations. Otherwise, validated machine-specific cutoff values have to be determined [23]. Opportunistic screening for osteoporosis becomes increasingly popular $[14,45]$. In contrast to numerous studies of opportunistic screening, where $\mathrm{HU}$ values in thoracic or lumbar vertebra were reported [33-35, 46-50], we used asynchronous calibration to obtain lumbar trabecular BMD. Synchronous
Table 3 Classifier performance of $\mathrm{BMD}_{\mathrm{QCT}}$ and DXA $T$-score for the prediction of incident vertebral fractures in ROC analysis

\begin{tabular}{|c|c|c|c|c|c|}
\hline \multirow[t]{3}{*}{ Classifier } & \multirow{3}{*}{$\begin{array}{l}\text { Area under the } \\
\text { ROC curve }(\mathrm{CI})\end{array}$} & \multicolumn{4}{|c|}{ BMD cutoff, $\mathrm{mg} / \mathrm{cm}^{3}$ (sensitivity) } \\
\hline & & \multicolumn{4}{|l|}{ Specificity } \\
\hline & & $75 \%$ & $81 \%$ & $88 \%$ & $94 \%$ \\
\hline BMD by QCT & $0.76(0.64-0.89)$ & $68.2(74 \%)$ & $79.6(59 \%)$ & $87.0(54 \%)$ & $104.8(37 \%)$ \\
\hline$T$-score by DXA & $0.63(0.48-0.78)$ & - & - & - & - \\
\hline
\end{tabular}

Statistically significant area under the ROC curve is in italics

CI, $95 \%$ confidence interval 


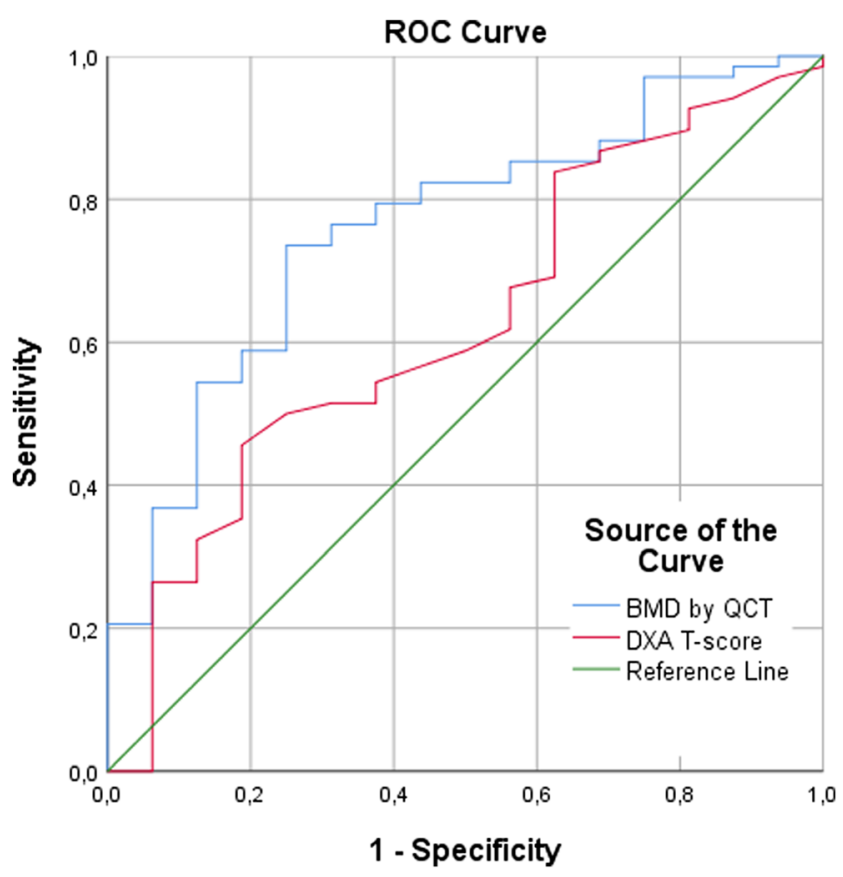

Fig. 5 Receiver-operating characteristics curves for predicting incident vertebral fractures by opportunistic QCT (BMD) and DXA (T-score)

calibration with an in-scan phantom as in conventional QCT can be replaced by asynchronous calibration as in the opportunistic setting, if scanner stability is maintained [23].

We performed asynchronous calibration of five different MDCT scanners to allow for opportunistic BMD screening in routine CT exams. In this opportunistic setting, the benefits of conventional QCT can be appreciated without its disadvantages of additional radiation and costs compared to DXA. We developed a protocol for dedicated calibration scans using the same parameters (tube voltage, average tube current, and reconstruction algorithm) and creating a similar geometrical setup of the scanned slice (anthropomorphic abdomen phantom with obesity extension rings and central inserts of known HA concentrations close to the position of the lumbar spine) as in routine scans. In case of the two already decommissioned MDCT scanners, we had to rely on routine scans with an in-plane density-reference phantom, yet we were able to exploit a huge number of scans averaging over a period of more than 4 years. We deemed the eccentric position of the in-plane phantom underneath the patient tolerable, as it was still close to the spine of the patient in supine position. This difference in position probably explains the additional intercept of approximately 15 units in the conversion equations of the two decommissioned Siemens scanners compared to the still operational Siemens scanner (Table 4).

Long-term scanner stability could be shown for three CT devices of two major manufacturers. Good short-term precision and low precision errors of intra-observer [51] and interobserver reproducibility [26] of asynchronous QCT have been shown before. How to correct for intravenous contrastenhanced scans in opportunistic QCT is still under debate $[14,45]$. We used correction offsets for arterial and portal venous contrast phase from a previous study [28], although these were calculated for a different CT scanner. These minor corrections might be negligible, because they did not seem to affect the accuracy of CT measurements [34] and the overall performance for predicting osteoporosis was similar [52], in previous studies. Moreover, in our data, contrast-enhanced scans were equally distributed between patients with and without incident vertebral fractures (Table 1), thus unlikely to bias the results.

There are limitations to this retrospective observational study. As only a limited cohort of mainly neurosurgical and oncologic patients were analyzed, the results might not be applicable to other patient populations. Additionally, all patients received both MDCT and DXA; thus, osteoporosis was already suggested. This may introduce a selection bias; however, this is exactly the patient population where omitting an additional DXA scan could save time and costs. Loss of follow-up is a possible confounding factor, though independent of the employed densitometric technique.

Table 4 HU-to-BMD conversion equations by asynchronous calibration and long-term stability for MDCT scanners used in this study

\begin{tabular}{|c|c|c|c|c|c|}
\hline \multirow[t]{2}{*}{ MDCT scanner } & \multirow{2}{*}{$\begin{array}{l}\text { Patients } \\
\text { (women) }\end{array}$} & \multicolumn{2}{|l|}{ HU-to-BMD conversion } & \multicolumn{2}{|c|}{ Long-term stability } \\
\hline & & Calibration phantom & $\begin{array}{l}\text { Conversion equations, } \\
\mathrm{BMD} \text { in } \mathrm{mg} / \mathrm{cm}^{3}\end{array}$ & $\begin{array}{l}\text { Observation } \\
\text { period, years }\end{array}$ & $\begin{array}{l}\text { Linear } \mathrm{HU} \text { change } \\
\text { per year }(\mathrm{CV})\end{array}$ \\
\hline Philips Brilliance 64 & $18(14)$ & QRM-BDC/3 & $\mathrm{BMD}=0.778 \times \mathrm{HU}-4.693$ & $\mathrm{n} / \mathrm{a}$ & $\mathrm{n} / \mathrm{a}$ \\
\hline Philips iCT 256 & $20(13)$ & QRM-Abdomen-Phantom & $\mathrm{BMD}=0.855 \times \mathrm{HU}+1.172$ & 5.33 & $-0.55(1.1 \%)$ \\
\hline Siemens Somatom Definition AS+ & $4(4)$ & QRM-Abdomen-Phantom & $\mathrm{BMD}=1.011 \times \mathrm{HU}-3.385$ & $\mathrm{n} / \mathrm{a}$ & $\mathrm{n} / \mathrm{a}$ \\
\hline Siemens Somatom Definition AS & $28(24)$ & Siemens Osteo* & $\mathrm{BMD}=0.985 \times \mathrm{HU}+15.516$ & 4.0 & $-2.29(1 \%)$ \\
\hline Siemens Somatom Sensation Cardiac 64 & $14(12)$ & Siemens Osteo* & $\mathrm{BMD}=0.971 \times \mathrm{HU}+13.249$ & 4.09 & $-0.81(0.7 \%)$ \\
\hline
\end{tabular}

The calibration Phantom marked with an asterisk $(*)$ was situated under the patient in the scanner couch during non-dedicated clinical CT scans (Fig. 2) and retrospectively used for asynchronous calibration. QRM-BDC/3, bone density calibration phantom with 3 rods of defined hydroxyapatite concentration; QRM-abdomen-phantom, anthropomorphic abdomen phantom with $400 \times 300 \mathrm{~mm}$ obesity extension ring and central insert with 4 rods of defined hydroxyapatite concentrations; $C V$, coefficient of variation of the standard error of the estimate 


\section{Conclusion}

Osteoporotic trabecular BMD of lumbar vertebrae assessed by opportunistic QCT was associated with an increased risk of incident vertebral fractures in mainly neurosurgical and oncologic patients aged 50 years and older. In contrast, $T$-scores derived from areal BMD by DXA had a less important effect on the fracture risk than age. Opportunistic screening in CT acquired for other purposes can improve the prediction of future vertebral fractures compared to dedicated DXA exams. The feasibility of pro- and retrospective asynchronous calibration with good long-term stability was shown for multiple MDCT scanners, allowing the scanner independent use of preestablished BMD cutoffs for the diagnosis of osteoporosis.

Funding This study has received funding by European Research Council (ERC) under the European Union's Horizon 2020 research and innovation program (grant agreement No 637164 - iBack - ERC-2014-STG).

\section{Compliance with ethical standards}

Guarantor The scientific guarantor of this publication is Jan S. Kirschke, Department of Neuroradiology, Klinikum rechts der Isar, Technische Universität München.

Conflict of interest The authors of this manuscript declare no relationships with any companies whose products or services may be related to the subject matter of the article.

Statistics and biometry One of the authors has significant statistical expertise.

No complex statistical methods were necessary for this paper.

Informed consent Written informed consent was waived by the Institutional Review Board.

Ethical approval Institutional Review Board approval was obtained.

\author{
Methodology \\ - retrospective \\ - observational \\ - performed at one institution
}

Open Access This article is distributed under the terms of the Creative Commons Attribution 4.0 International License (http:// creativecommons.org/licenses/by/4.0/), which permits unrestricted use, distribution, and reproduction in any medium, provided you give appropriate credit to the original author(s) and the source, provide a link to the Creative Commons license, and indicate if changes were made.

Publisher's note Springer Nature remains neutral with regard to jurisdictional claims in published maps and institutional affiliations.

\section{References}

1. (1993) Consensus development conference: diagnosis, prophylaxis, and treatment of osteoporosis. Am J Med 94:646-650. https://doi. org/10.1016/0002-9343(93)90218-E
2. Häussler B, Gothe H, Göl D, Glaeske G, Pientka L, Felsenberg D (2007) Epidemiology, treatment and costs of osteoporosis in Germany-the BoneEVA study. Osteoporos Int 18:77-84. https://doi. org/10.1007/s00198-006-0206-y

3. Hernlund E, Svedbom A, Ivergård M et al (2013) Osteoporosis in the European Union: medical management, epidemiology and economic burden. A report prepared in collaboration with the International Osteoporosis Foundation (IOF) and the European Federation of Pharmaceutical Industry Associations (EFPIA). Arch Osteoporos 8:136. https://doi.org/10.1007/s11657-013-0136-1

4. Cosman F, de Beur SJ, LeBoff MS et al (2014) Clinician's guide to prevention and treatment of osteoporosis. Osteoporos Int 25:23592381. https://doi.org/10.1007/s00198-014-2794-2

5. Khosla S, Shane E (2016) A crisis in the treatment of osteoporosis. J Bone Miner Res 31:1485-1487. https://doi.org/10.1002/jbmr.2888

6. Zhang J, Delzell E, Zhao H et al (2012) Central DXA utilization shifts from office-based to hospital-based settings among Medicare beneficiaries in the wake of reimbursement changes. J Bone Miner Res 27:858-864. https://doi.org/10.1002/jbmr.1534

7. Curtis JR, Carbone L, Cheng H et al (2008) Longitudinal trends in use of bone mass measurement among older Americans, 1999-2005. J Bone Miner Res 23:1061-1067. https://doi.org/10.1359/jbmr.080232

8. NIH Consensus Development Panel on Osteoporosis Prevention, Diagnosis, and Therapy (2001) Osteoporosis prevention, diagnosis, and therapy. JAMA 285:785-795

9. Jain RK, Vokes T (2017) Dual-energy X-ray absorptiometry. J Clin Densitom 20:291-303. https://doi.org/10.1016/j.jocd.2017.06.014

10. Kanis JA (1994) Assessment of fracture risk and its application to screening for postmenopausal osteoporosis: synopsis of a WHO report. WHO Study Group. Osteoporos Int 4:368-381

11. Schuit SC, van der Klift M, Weel AE et al (2004) Fracture incidence and association with bone mineral density in elderly men and women: the Rotterdam study. Bone 34:195-202

12. Engelke K, Adams JE, Armbrecht G et al (2008) Clinical use of quantitative computed tomography and peripheral quantitative computed tomography in the management of osteoporosis in adults: the 2007 ISCD Official Positions. J Clin Densitom 11:123-162. https://doi.org/10.1016/j.jocd.2007.12.010

13. Papadakis AE, Karantanas AH, Papadokostakis G, Petinellis E, Damilakis J (2009) Can abdominal multi-detector CT diagnose spinal osteoporosis? Eur Radiol 19:172-176. https://doi.org/10. 1007/s00330-008-1099-2

14. Engelke K (2017) Quantitative computed tomography-current status and new developments. J Clin Densitom 20:309-321. https:// doi.org/10.1016/j.jocd.2017.06.017

15. Guglielmi G, Grimston SK, Fischer KC, Pacifici R (1994) Osteoporosis: diagnosis with lateral and posteroanterior dual $\mathrm{x}$ ray absorptiometry compared with quantitative CT. Radiology 192:845-850. https://doi.org/10.1148/radiology.192.3.8058958

16. Lafferty FW, Rowland DY (1996) Correlations of dual-energy Xray absorptiometry, quantitative computed tomography, and single photon absorptiometry with spinal and non-spinal fractures. Osteoporos Int 6:407-415

17. Rehman Q, Lang T, Modin G, Lane NE (2002) Quantitative computed tomography of the lumbar spine, not dual x-ray absorptiometry, is an independent predictor of prevalent vertebral fractures in postmenopausal women with osteopenia receiving long-term glucocorticoid and hormone-replacement therapy. Arthritis Rheum 46: 1292-1297. https://doi.org/10.1002/art.10277

18. Yu W, Glüer CC, Grampp S et al (1995) Spinal bone mineral assessment in postmenopausal women: a comparison between dual $\mathrm{X}$-ray absorptiometry and quantitative computed tomography. Osteoporos Int 5:433-439

19. Lee SJ, Graffy PM, Zea RD, Ziemlewicz TJ, Pickhardt PJ (2018) Future osteoporotic fracture risk related to lumbar vertebral 
trabecular attenuation measured at routine body CT. J Bone Miner Res 33:860-867. https://doi.org/10.1002/jbmr.3383

20. Genant HK, Wu CY, van Kuijk C, Nevitt MC (1993) Vertebral fracture assessment using a semiquantitative technique. J Bone Miner Res 8:1137-1148. https://doi.org/10.1002/jbmr.5650080915

21. Hedderich DM, Maegerlein C, Baum T et al (2018) Differentiation of acute/subacute versus old vertebral fractures in MDCT - is MRI always needed? World Neurosurg. https://doi.org/10.1016/j.wneu.2018.10.121

22. Piazzolla A, Solarino G, Lamartina C et al (2015) Vertebral bone marrow edema (VBME) in conservatively treated acute vertebral compression fractures (VCFs): evolution and clinical correlations. Spine (Phila Pa 1976) 40:E842-E848. https://doi. org/10.1097/BRS.0000000000000973

23. Shepherd JA, Schousboe JT, Broy SB, Engelke K, Leslie WD (2015) Executive summary of the 2015 ISCD position development conference on advanced measures from DXA and QCT: fracture prediction beyond BMD. J Clin Densitom 18:274-286. https://doi. org/10.1016/j.jocd.2015.06.013

24. Lewiecki EM, Binkley N, Morgan SL et al (2016) Best practices for dual-energy X-ray absorptiometry measurement and reporting: International Society for Clinical Densitometry Guidance. J Clin Densitom 19:127-140. https://doi.org/10.1016/j.jocd.2016.03.003

25. Bauer JS, Müller D, Ambekar A et al (2006) Detection of osteoporotic vertebral fractures using multidetector $\mathrm{CT}$. Osteoporos Int 17: 608-615. https://doi.org/10.1007/s00198-005-0023-8

26. Brown JK, Timm W, Bodeen G et al (2017) Asynchronously calibrated quantitative bone densitometry. J Clin Densitom 20:216225. https://doi.org/10.1016/j.jocd.2015.11.001

27. Baum T, Müller D, Dobritz M, Rummeny EJ, Link TM, Bauer JS (2011) BMD measurements of the spine derived from sagittal reformations of contrast-enhanced MDCT without dedicated software. Eur J Radiol 80:e140-e145. https://doi.org/10.1016/j.ejrad.2010. 08.034

28. Kaesmacher J, Liebl H, Baum T, Kirschke JS (2017) Bone mineral density estimations from routine multidetector computed tomography: a comparative study of contrast and calibration effects. J Comput Assist Tomogr 41:217-223. https://doi.org/10.1097/RCT. 0000000000000518

29. American College of Radiology (2018) ACR-SPR-SSR practice parameter for the performance of musculoskeletal quantitative computed tomography (QCT). American College of Radiology, Reston. Available via https://www.acr.org/-/media/ACR/Files/PracticeParameters/QCT.pdf?la=en. Accessed 7 Nov 2018

30. Glüer CC, Blake G, Lu Y, Blunt BA, Jergas M, Genant HK (1995) Accurate assessment of precision errors: how to measure the reproducibility of bone densitometry techniques. Osteoporos Int 5:262270

31. Grampp S, Genant HK, Mathur A et al (1997) Comparisons of noninvasive bone mineral measurements in assessing age-related loss, fracture discrimination, and diagnostic classification. J Bone Miner Res 12:697-711. https://doi.org/10.1359/jbmr.1997.12.5. 697

32. Ito M, Hayashi K, Ishida $Y$ et al (1997) Discrimination of spinal fracture with various bone mineral measurements. Calcif Tissue Int 60:11-15

33. Alacreu E, Moratal D, Arana E (2017) Opportunistic screening for osteoporosis by routine CT in Southern Europe. Osteoporos Int 28: 983-990. https://doi.org/10.1007/s00198-016-3804-3

34. Pickhardt PJ, Pooler BD, Lauder T et al (2013) Opportunistic screening for osteoporosis using abdominal computed tomography scans obtained for other indications. Ann Intern Med 158:588-595. https://doi.org/10.7326/0003-4819-158-8-201304160-00003

35. Marinova M, Edon B, Wolter K, Katsimbari B, Schild HH, Strunk HM (2015) Use of routine thoracic and abdominal computed tomography scans for assessing bone mineral density and detecting osteoporosis. Curr Med Res Opin 31:1871-1881. https://doi.org/ 10.1185/03007995.2015.1074892

36. Reid IR, Evans MC, Ames R, Wattie DJ (1991) The influence of osteophytes and aortic calcification on spinal mineral density in postmenopausal women. J Clin Endocrinol Metab 72:1372-1374. https://doi.org/10.1210/jcem-72-6-1372

37. Orwoll ES, Oviatt SK, Mann T (1990) The impact of osteophytic and vascular calcifications on vertebral mineral density measurements in men. J Clin Endocrinol Metab 70:1202-1207. https://doi. org/10.1210/jcem-70-4-1202

38. Yu W, Glüer CC, Fuerst T et al (1995) Influence of degenerative joint disease on spinal bone mineral measurements in postmenopausal women. Calcif Tissue Int 57:169-174

39. Fidler JL, Murthy NS, Khosla S et al (2016) Comprehensive assessment of osteoporosis and bone fragility with CT colonography. Radiology 278:172-180. https://doi.org/10.1148/radiol. 2015141984

40. Li N, Li XM, Xu L, Sun WJ, Cheng XG, Tian W (2013) Comparison of QCT and DXA: osteoporosis detection rates in postmenopausal women. Int J Endocrinol 2013:895474. https:// doi.org/10.1155/2013/895474

41. Wang X, Sanyal A, Cawthon PM et al (2012) Prediction of new clinical vertebral fractures in elderly men using finite element analysis of CT scans. J Bone Miner Res 27:808-816. https://doi.org/10. 1002/jbmr.1539

42. Chalhoub D, Orwoll ES, Cawthon PM et al (2016) Areal and volumetric bone mineral density and risk of multiple types of fracture in older men. Bone 92:100-106. https://doi.org/10.1016/j.bone. 2016.08.014

43. Kopperdahl DL, Aspelund T, Hoffmann PF et al (2014) Assessment of incident spine and hip fractures in women and men using finite element analysis of CT scans. J Bone Miner Res 29:570-580. https://doi.org/10.1002/jbmr.2069

44. Felsenberg D, Gowin W (1999) Bone densitometry by dual energy methods. Radiologe 39:186-193. https://doi.org/10.1007/ s001170050495

45. Engelke K, Lang T, Khosla S et al (2015) Clinical use of quantitative computed tomography-based advanced techniques in the management of osteoporosis in adults: the 2015 ISCD Official Positions-Part III. J Clin Densitom 18:393-407. https://doi.org/10. 1016/j.jocd.2015.06.010

46. Buckens CF, van der Graaf Y, Verkooijen HM et al (2015) Osteoporosis markers on low-dose lung cancer screening chest computed tomography scans predict all-cause mortality. Eur Radiol 25:132-139. https://doi.org/10.1007/s00330-0143361-0

47. Fang J, Franconeri A, Boos J et al (2018) Opportunistic bone density measurement on abdomen and pelvis computed tomography to predict fracture risk in women aged 50 to 64 years without osteoporosis risk factors. J Comput Assist Tomogr 42: 798-806. https://doi.org/10.1097/RCT.0000000000000744

48. Lee SJ, Binkley N, Lubner MG, Bruce RJ, Ziemlewicz TJ, Pickhardt PJ (2016) Opportunistic screening for osteoporosis using the sagittal reconstruction from routine abdominal CT for combined assessment of vertebral fractures and density. Osteoporos Int 27:1131-1136. https://doi.org/10.1007/s00198015-3318-4

49. Li YL, Wong KH, Law MW et al (2018) Opportunistic screening for osteoporosis in abdominal computed tomography for Chinese population. Arch Osteoporos 13:76. https://doi.org/10.1007/ s11657-018-0492-y

50. Rebello D, Anjelly D, Grand DJ et al (2018) Opportunistic screening for bone disease using abdominal CT scans obtained for other reasons in newly diagnosed IBD patients. Osteoporos Int 29:13591366. https://doi.org/10.1007/s00198-018-4444-6 
51. Wang L, Su Y, Wang Q et al (2017) Validation of asynchronous quantitative bone densitometry of the spine: accuracy, short-term reproducibility, and a comparison with conventional quantitative computed tomography. Sci Rep 7:6284. https://doi.org/10.1038/ s41598-017-06608-y
52. Pickhardt PJ, Lauder T, Pooler BD et al (2016) Effect of IV contrast on lumbar trabecular attenuation at routine abdominal CT: correlation with DXA and implications for opportunistic osteoporosis screening. Osteoporos Int 27:147-152. https://doi.org/10.1007/ s00198-015-3224-9 